Article

\title{
Evaluation of Urbanization Dynamics and its Impacts on Surface Heat Islands: A Case Study of Beijing, China
}

\author{
Wei Chen, Yao Zhang, Chongyu Pengwang and Weijun Gao *
}

Faculty of Environment Engineering, The University of Kitakyushu, Kitakyushu 808-0135, Japan; chenwei19910415@gmail.com (W.C.); yaoyao661025@163.com (Y.Z.); pengwcy@gmail.com (C.P.)

* Correspondence: weijun@kitakyu-u.ac.jp; Tel.: +81-93-695-3234

Academic Editors: Bailang Yu, Yuyu Zhou, Chunyang He, Xiaofeng Li, James Campbell and Prasad S. Thenkabail

Received: 23 January 2017; Accepted: 5 May 2017; Published: 7 May 2017

\begin{abstract}
As the capital of China, Beijing has experienced a continued and rapid urbanization process in the past few decades. One of the key environmental impacts of rapid urbanization is the effect of urban heat island (UHI). The objective of this study was to estimate the urbanization indexes of Beijing from 1992 to 2013 based on the stable nighttime light (NTL) data derived from the Defense Meteorological Satellite Program's Operational Line Scanner System (DMSP/OLS), which has became a widely used remote sensing database after decades of development. The annual average value nighttime light Digital Number (NTL-DN), and the total lit number and urban area proportion within Beijing's boundary were calculated and compared with social-economic statistics parameters to estimate the correlation between them. Four Landsat thematic mapper (TM) images acquired in 1995 and 2009 were applied to estimate the normalized difference vegetation index (NDVI) and normalized land surface temperature $\left(\mathrm{LST}_{\text {nor }}\right)$, and spatial correlation analysis was then carried out to investigate the relationship between the urbanization level and NDVI and $\mathrm{LST}_{\text {nor }}$. Our results showed a strong negative linear relationship between the NTL-DN value and NDVI; however, in contrast, a strong positive linear relationship between existed between the NTL-DN value and $\mathrm{LST}_{\text {nor. }}$. By conducting a spatial comparison analysis of 1995 and 2009, the vegetation coverage change and surface temperature difference were calculated and compared with the NTL-DN difference. Our result revealed that the regions of fast urbanization resulted in a decrease of NDVI and increase of $\mathrm{LST}_{\text {nor }}$. In addition, choropleth maps showing the spatial pattern of urban heat island zones were produced based on different temperatures, and the analysis result indicated that the spatial distribution of surface temperature was closely related with the NTL-DN and NDVI. These findings are helpful for understanding the urbanization process as well as urban ecology, which both have significant implications for urban planning and minimize the potential environmental impacts of urbanization in Beijing.
\end{abstract}

Keywords: urbanization; DMSP/OLS; Landsat; land surface temperature; NDVI; Beijing

\section{Introduction}

Currently, more than $50 \%$ of the world's population lives in urban areas, with this proportion likely to keep increasing in developing countries. Urbanization is taking place at a spectacular rate worldwide, particularly in China during the past few decades. With continuous rural-urban migration and urban expansion, more megacities are continuously emerging in China [1,2]. Along with rapid urbanization and modernization, some environmental issues, such as water pollution [3], air pollution [4], greenhouse gas emissions [5], and enhanced urban heat islands [6], are arising in 
many Chinese cities. Therefore, to have a comprehensive understanding of the process of urbanization and to evaluate its environmental influence, it is necessary and indispensable to monitor and analyze the dynamics of urbanization in China [7]. As the capital city of China, Beijing has experienced rapid urbanization, industrialization and modernization, therefore, analyzing the urbanization process of Beijing has significant meaning, which would be conductive to studies of other cities of China.

In recent years, satellite observations have been widely used in urban studies across the world, one of which is the Defense Meteorological Satellite Program's Operational Line Scanner System (DMSP/OLS). The DMSP/OLS dataset, which was originally used to monitor the global distribution of clouds and cloud top temperatures in the 1970s, was not widely noticed until 1992. During the last two decades, DMSP/OLS nighttime light (NTL) images have been developed and extensively applied to urban studies. These studies included mapping urban areas [8], and detecting spatial urban sprawl trends [9]; in addition, NTL imagery is regarded as a primary data source to estimate the variation and development of urban population and population density $[10,11]$, detect socio-economic activity and dynamics [12], measure impact on energy and electricity consumption [13], and evaluate gas emissions [14]. The spatial-temporal dynamics of urbanization based on DMSP/OLS of Chinese cities was characterized and estimated by integrating social-economic statistics [7,15], which provided timely and spatially explicit metrics of the urbanization processes. Numerous studies put the DMSP/OLS database into use as a spatial reflection of the urbanization level; nevertheless, there have been few studies focused on the integration of spatial distribution dynamics with the thermal environment and heat island effects.

Urbanization profoundly influences biodiversity and ecosystem function, as well as local and regional climate and quality of life [16]. One of the more serious impacts of urbanization is the urban heat island (UHI) effect, which indicates that an urban area is significantly warmer than its surrounding rural areas due to artificial land cover and anthropogenic heat. UHI is an important issue for urban planning and environment improvement as it has several negative impacts on an urban environment. Traditionally, UHI is observed from air temperature measurements within the urban area and the surrounding rural areas; however, more recent UHI studies are based on land surface temperature (LST) from remote sensors because of high spatial and temporal resolution, free availability and easy access. Therefore, remote sensing based UHI has been used as the surface urban heat island (SUHI) $[17,18]$. Particularly, the Moderate Resolution Imaging Spectroradiometer (MODIS) on board Terra and Aqua, which provides daily LST data with high temporal (four times per day) and relatively high spatial resolution $(1 \mathrm{~km})$, has been widely applied for monitoring UHI $[19,20]$. On the other hand, with a high spatial resolution of 30-120 m, Landsat TM/ETM+/OLI data has been used to estimate the LST for SUHI study in recent years [21-23], and mature and applicable technology of estimating LST was developed using the Landsat products. In addition, as vegetation transpiration mitigates the effect of UHI, numerous studies have focused on understanding the relationship between LST and the normalized difference vegetation index (NDVI) [24-26]. These studies found that the NDVI-LST relationship was more suitable for the analysis of UHI in summer and early autumn [23]. However, a few studies focused on the UHI analysis of the integrated application of multi-sensors, such as the combination of thermal remote sensing and DMSP/OLS. Liao et al. [19] analyzed the impact of energy consumption on the surface urban heat island in China's 32 major cities by using MODIS LST data and DMSP/OLS nighttime light images. In this study, to explore the feasibility of the combinative applications of Landsat products and DMSP/OLS NTL images, the Landsat based NDVI and LST maps were applied to establish the spatial correlation with DMSP/OLS NTL imagery to estimate the urbanization impact on the SUHI.

This study was composed of two parts. First, a reliable method of estimating the urbanization process based on DMSP/OLS NTL data was carried out to analyze the dynamics of urbanization levels in Beijing from 1992-2013. In this part, the DMSP/OLS NTL was applied to calculate the average NTL-DN, the lit number, and the extraction of urban area proportion for further correlation analysis with the social-economic statistics. In the second part, the study explored the use of multi-sensor 
remote-sensing data, including the integrated application of DMSP/OLS NTL imagery and Landsat TM products, and estimated the interrelationship between the spatial distribution of urbanization level

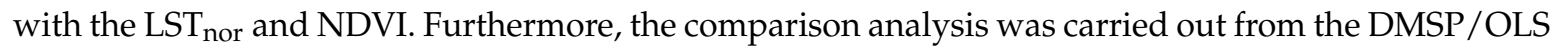

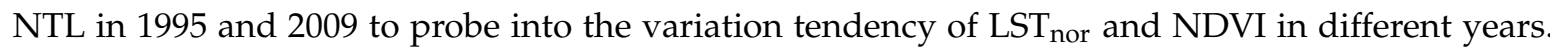
In addition, the target region was divided into four categories based on temperature distribution character in each year, and statistical and comparative analysis among the different urban heat island zones were used to explore the correlation between urban heat island effects, and the NTL and NDVI.

\section{Data and Methods}

As the capital of China, Beijing is well known worldwide as a famous ancient city and an international metropolis, which is located at $39^{\circ} 56^{\prime} \mathrm{N}$ and $116^{\circ} 20^{\prime}$ E. Figure 1 shows the geographic location of the study area (Figure 1a) and administrative districts of the Beijing city (Figure 1b). As the political, cultural and economic center of the People's Republic of China, Beijing city covers an area of $16,808 \mathrm{~km}^{2}$. In terms of topography, two-thirds of Beijing are mountainous areas where the city is surrounded by mountains from all sides, except for the southeast of the city, where a plain slopes slightly to the Bohai Rim. Beijing city is characterized by a warm temperature zone and has a typical continental monsoon climate with four distinct seasons, including a hot and rainy summer and a cold and dry winter.

(a)

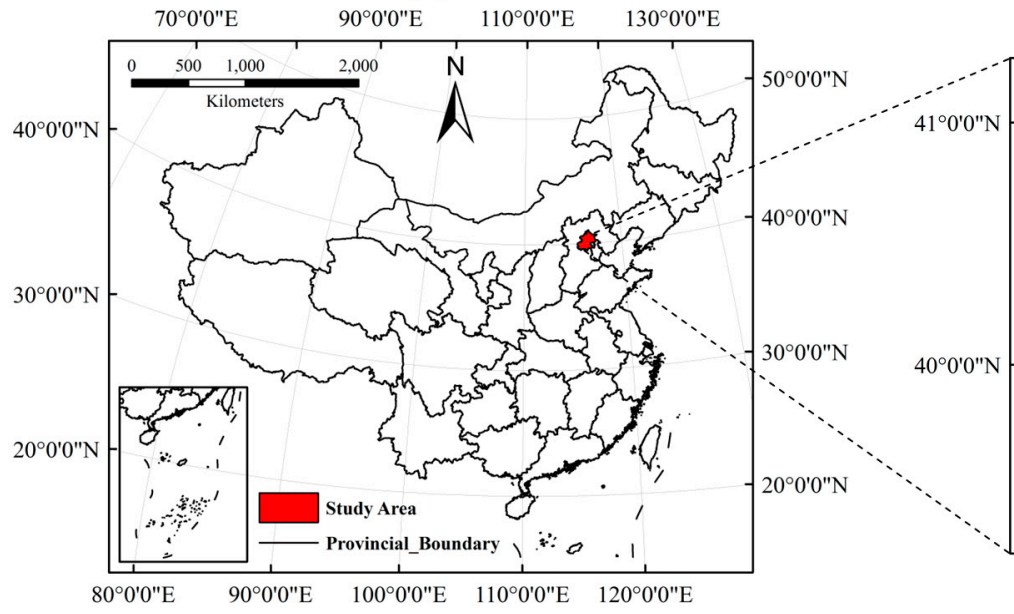

(a) (b)

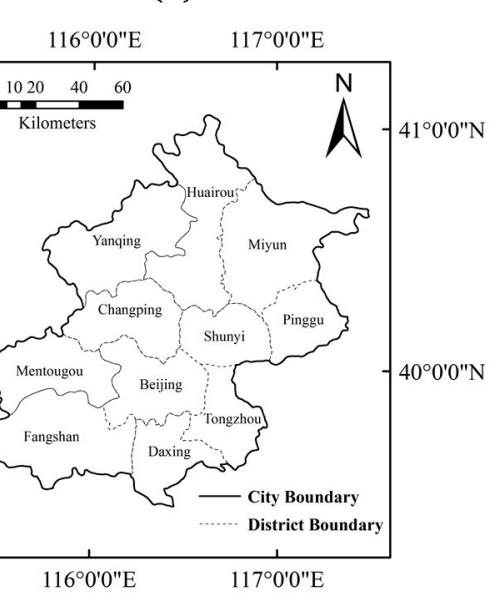

(b)

Figure 1. Location map of the study area and Beijing city districts (a) Geographic location of the study area; (b) Administrative districts of the Beijing city.

After 1949 planners were concerned with defining the city as a political and cultural center, the city has been experiencing unprecedented urbanization over the last several decades [27]. The rapid economic growth of Beijing is not only associated with the large-scale migration of population [28], but is also connected with many environmental consequences including UHI effects. Urbanization is a quite complicated phenomenon, with many driving forces responsible for the urbanization of a city. As the capital city of China, the situation in Beijing is more complicated, and it is important to evaluate the transformation processes to support policy making for environmental management and urban planning. Thus, investigating the spatial and temporal urbanization situation and understanding the UHI development situation in Beijing is crucial for the sustainable development of the city in the context of policy making. For the present study, the following methodology was adopted, which involved the collection of social-economic statistics, satellite data collection, preprocessing of the remote sensing imagery, preparation of NDVI maps, retrieval of $\mathrm{LST}_{\text {nor }}$ maps, construction of choropleth maps of 
urban heat island zones and correlation analysis studies. The data sets used in this research are shown in Table 1 below.

Table 1. Data sets used in this research.

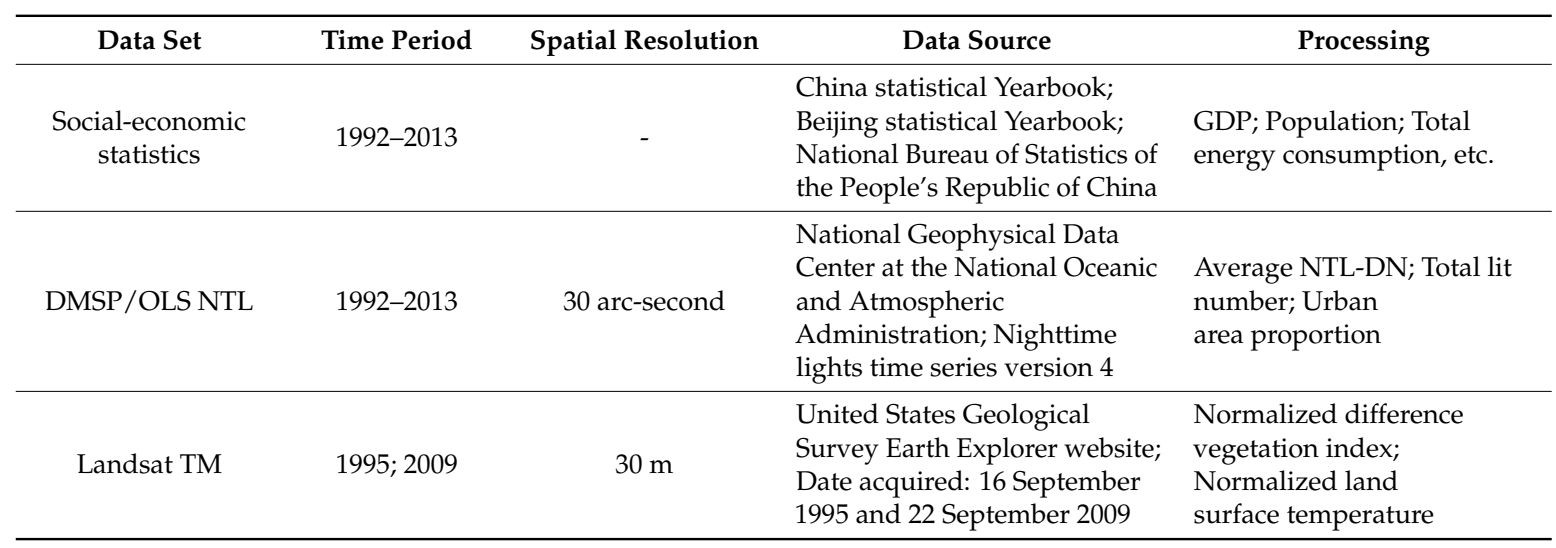

\subsection{Social-Economic Statistics Collection}

Social-economic statistics of Beijing were obtained from the China Statistical Yearbook, the Beijing Statistical Yearbook and the National Bureau of Statistics of the People's Republic of China [29]. The indicators were primarily selected according to the following general selection criterion: (1) choose the most cited indicators; (2) cover the components of urbanization processing and (3) choose the simplest indicators to facilitate indicators. Permanent population and gross domestic product (GDP) were selected as representative indicators of demographic and economic, in addition, these two indicators were the most widely used socio-economic indexes to establish the relationship with the urbanization processes based on DMSP/OLS NTL, which have been estimated by previous researchers $[9,10]$. Moreover, studies have confirmed that energy consumption and electricity consumption have close relationships with DMSP/OLS NTL based urbanization estimating [13,30]. Besides, popularization and promotion of natural gas in some ways could reflect the development of energy industry structure in the city, there is also little research on establishing relationship with DMSP/OLS NTL, as a result, total consumption of natural gas was selected. Freight traffic plays an important role during the development of a city, length of highways and the total number of civil motor vehicles were chose to reflect the freight traffic development in Beijing. Therefore, the data collection includes seven annual indexes: GDP; permanent population; total energy consumption; total consumption of natural gas; the total electricity consumption; length of highways; and the total number of civil motor vehicles.

\subsection{Preprocessing of DMSP/OLS NTL Imagery}

Nighttime stable light data from 1992-2013 were obtained from the National Oceanic and Atmospheric Administration (NOAA)/National Geophysical Data Center (NGDC) web site and included data from six individual sensors: F10 (1992-1994), F12 (1994-1999), F14 (1997-2003), F15 (2000-2007), F16 (2004-2009), F18 (2010-2013) [31]. Annual composites were produced for each satellite using the highest-quality of collected data. These images are grid-based compositions with a 0-63 digital number (DN) and a 30 arc-second (approximately $1 \mathrm{~km}$ at the equator) spatial resolution for pixels.

\subsubsection{Inter-Calibration}

Due to the lack of on-board calibration, the NTL-DN among annual DMSP/OLS NTL images were not compatible. To improve the comparability of NLT data in Beijing from the period 1992-2013, a second order regression model proposed by Elvidge et al. [32] was utilized to generate an 
inter-calibrated DMSP/OLS NTL time series and was matched with the composite of F12 in 1999 to minimize the effects of variation among sensors. On this basis, the NTL series can be adjusted to the same radiometric baseline [32]. As the model was empirically developed using the region with little NTL change as the reference data, by analyzing the socio-economic characteristics based on GDP and built-up area data for Chinese cities from 1992-2013, Yichun City in Heilongjiang Province, China was selected as the reference region. A set of annual composites were compared with F121999 for the city of Yichun using the regression model below. In Equation (1), DN is the original NTL-DN value, $D N_{\text {calibrated }}$ is the inter-calibrated, a, b, and c are coefficients.

$$
D N_{\text {calibrated }}=a \times D N^{2}+b \times D N+c,
$$

\subsubsection{Intra-Annual Composition and Inter-Annual Correction}

To make full use of the data collected from two satellites for the same year and to remove the intra-annual unstable lit pixels, a systematic correction method proposed by Liu et al. [33] was applied as intra-annual composition. The composition produced one intra-annual composite for the year with two NTL images from the same year.

In addition, to make sure the NTL based urban dynamics matched the actual process and to remove the discrepancies in the multi-year dataset, an assumption was proposed that the area would develop continuously, leading to an only grow trend of NTL-DN value over time [31]. The inter-annual correction carried out was applied to each NTL image [33].

\subsubsection{Calculation of DMSP/OLS NTL Indicators}

Our method for analyzing the dynamics of urbanization processes in Beijing includes three main steps: calculating the annual average NTL-DN; counting the total number of lit pixels; calculation the urban area proportion.

1. Calculation of the annual average NTL-DN

In this study, we used the annual average NTL-DN as one of three indicators to estimate the annual degree of urbanization in Beijing. The annual average NTL-DN was defined as the annual average NTL-DN value of all pixels located in the administrative districts. By taking the pixels located at the boundaries into consideration, the DN value of each pixel was multiplied by the area that it located. In addition, the calculation was made after the preprocessing of the DMSP/OLS NTL imagery, and can be illustrated by Equation (2). In the equation, $D N_{\text {pixel }}$ and $A r e a_{\text {pixel }}$ are the NTL-DN value and the trapezoid area of each pixel, Area $_{\text {sum }}$ is the sum of the area within the administrative boundary.

$$
\text { Annual average NTL }-\mathrm{DN}=\frac{\sum D N_{\text {pixel }} \times \text { Area }_{\text {pixel }}}{\text { Area }_{\text {sum }}},
$$

\section{Counting the total number of lit pixels}

The total number of lit pixels explained the overall level of how many pixels were lit within the scope of the study region. The total number of pixels derived from the satellite within Beijing city was 24,991, and after inter-annual correlation, the total number of pixels greater than 0 in each year from each satellite was counted. For the year with two satellite datasets, the pixel was counted sonly if it was lit in either dataset.

\section{Urban area proportion}

For the extraction of lit urban area, we adopted the threshold technique developed by Henderson et al. [34]. Furthermore, the optimal thresholds for extraction were determined based on the method provided by Gao et al. [35]. As Beijing is in the Northern Coastal China (NCC) region, the thresholds of NCC were exploited for the extraction. 


\subsection{Preprocessing of Landsat TM Images}

Four Landsat 5 TM images were collected on 16 September 1995 (Landsat Scene ID: LT51230321995259HAJ00 and LT51230331995259HAJ00) and 22 September 2009 (Landsat Scene ID: LT51230322009265IKR00 and LT51230332009265IKR00) from the United States Geological Survey (USGS) Earth Explorer website [36]. The Landsat TM images were all acquired under clear sky conditions. The images were further rectified to the Universal Transverse Mercator projection system (datum WGS84, UTM zone N50). The Landsat TM products were composed of seven bands, six of them in the visible and near infrared, with only one band located in the thermal infrared region.

To cover the whole administrative region of Beijing, two images were merged together as a preliminary step. The next step consisted of the calculation of at-sensor spectral radiance in converting image data into a physically meaningful common radiometric scale. Equation (3) was applied to convert the digital numbers for both reflective and thermal bands to at-sensor radiance [37,38]:

$$
\mathrm{L}_{\lambda}=\frac{L M A X_{\lambda}-L M I N_{\lambda}}{Q_{\text {calmax }}-Q_{\text {calmin }}} \times\left(Q_{\text {cal }}-Q_{\text {calmin }}\right)+\operatorname{LMIN}_{\lambda}
$$

where $L_{\lambda}$ is the spectral radiance at the sensor's aperture in $\mathrm{W} /\left(\mathrm{m}^{2} \mathrm{sr} \mu \mathrm{m}\right) ; Q_{c a l}$ is the quantized calibrated pixel value in $\mathrm{DN} ; Q_{\text {calmin }}$ is the minimum quantized calibrated pixel value corresponding to $L M I N_{\lambda} ; Q_{\text {calmax }}$ is the maximum quantized calibrated pixel value corresponding to $L M A X_{\lambda} ; L M I N_{\lambda}$ is the spectral radiance that is scaled to $Q_{\text {calmin }}$ in $\mathrm{W} /\left(\mathrm{m}^{2} \cdot \mathrm{sr} \cdot \mu \mathrm{m}\right)$; and $L M A X_{\lambda}$ is the spectral radiance that is scaled to $Q_{\text {calmax }}$ in $\mathrm{W} /\left(\mathrm{m}^{2} \cdot \mathrm{sr} \cdot \mu \mathrm{m}\right)$.

To reduce the between-scene variability of relatively clear Landsat scenes for all bands, we adopted top-of-atmosphere (TOA) reflectance to correct the cosine effect of solar zenith angles and solar irradiance [37]. The combined surface and atmospheric reflectance was computed as per Equation (4) [38]:

$$
\rho_{\lambda}=\frac{\pi \cdot L_{\lambda} \cdot d^{2}}{\operatorname{ESUN}_{\lambda} \cdot \cos \theta_{S}}
$$

where $\rho_{\lambda}$ is the unitless planetary reflectance; $L_{\lambda}$ is the spectral radiance at the sensor's aperture in $\mathrm{W} /\left(\mathrm{m}^{2} \cdot \mathrm{sr} \cdot \mu \mathrm{m}\right) ; d$ is the earth-sun distance (in astronomical units); ESUN $N_{\lambda}$ is the mean solar exo-atmospheric irradiance in $\mathrm{W} /\left(\mathrm{m}^{2} \cdot \mu \mathrm{m}\right)$; and $\theta_{s}$ is the solar zenith angle in degrees.

\subsubsection{Normalized Difference Vegetation Index Calculation}

The NDVI index is a measure of the amount and vigor of vegetation at the surface. As vegetation reflects well in the near infrared part of the spectrum, NDVI has become a simple graphical indicator to assess the vegetation coverage of the target. Numerous studies have focused on understanding the relationship between LST and NDVI [26] and concluded that a close relationship between them existed. For this study, to explore the relationship between vegetation coverage and LST and DMSP/OLS based urbanization level, the Landsat image based NDVI was calculated as the reflection of the vegetation coverage. Data supplied by Band 3 and Band 4 can be used to construct this vegetation index according to the following equation:

$$
N D V I=\frac{\text { Band } 4-\text { Band } 3}{\text { Band } 4+\text { Band } 3}
$$

\subsubsection{Estimating Land Surface Temperature}

The procedure described by Weng et al. [39] was adopted for the retrieval of LST. The thermal infrared band (Band 6) was utilized to map LST, and the processing was composed of four parts:

1. First, converting spectral radiance to at-sensor spectral, which is a more physically useful variable, and was calculated by Equation (3); 
2. Second, to converting the result of Step 1 to at-sensor brightness temperature, this proceeded under the assumption that the earth's surface is a black body, and using the formula below [38];

$$
T_{B}=\frac{K_{2}}{\ln \left(\frac{K_{1}}{L_{\lambda}}+1\right)},
$$

where $T_{B}$ is the effective at sensor brightness temperature in Kelvin; $\mathrm{L}_{\lambda}$ is the spectral radiance at the sensor's aperture in $\mathrm{W} /\left(\mathrm{m}^{2} \cdot \mathrm{sr} \cdot \mu \mathrm{m}\right) ; K_{1}$ and $K_{2}$ are the calibration constant for Landsat TM; $K_{1}$ is $607.76 \mathrm{~W} /\left(\mathrm{m}^{2} \cdot \mathrm{sr} \cdot \mu \mathrm{m}\right)$; and $K_{2}$ is $1260.56 \mathrm{~K}$.

3. Third, the obtained brightness temperatures were referenced to a black body, which differ from the properties of real objects. Correction for spectral emissivity $(\varepsilon)$ is a must and the emissivity corrected surface temperature was computed as follows [40]:

$$
T_{S}=\frac{T_{B}}{1+\left(\lambda \times T_{B} / \alpha\right) \ln \varepsilon},
$$

where $T_{S}$ is the surface radiant temperature in Kelvin (k); $T_{B}$ is the effective at sensor brightness temperature in Kelvin; $\lambda$ is the wavelength of emitted radiance, herein, $\lambda=11.5 \mu \mathrm{m} \mathrm{[41];}$ $\alpha=h c / b\left(1.438 \times 10^{-2} \mathrm{~m} \cdot \mathrm{K}\right) ; b$ is the Bolzmann constant $\left(1.38 \times 10^{-23} \mathrm{~J} / \mathrm{K}\right) ; h$ is Plank's constant $\left(6.626 \times 10^{-34} \mathrm{Js}\right) ; c$ is the velocity of light $\left(2.998 \times 10^{8} \mathrm{~m} / \mathrm{s}\right)$; and $\varepsilon$ is the surface emissivity which can be calculated from NDVI [42,43], the estimation was shown in Table 2 below.

As the surface radiant temperature is in Kelvin, which differs from the commonly used centigrade, the LST in Celsius was calculated by adding the absolute zero (approximately $-273.15^{\circ} \mathrm{C}$ ) [44].

4. Finally, to ensure that the images from different years were comparable, normalized land surface temperature was put forward, which quantified the LST range from $0-1$. The calculation required the equation below:

$$
L S T_{\text {nor }}=\frac{L S T-L S T_{\text {min }}}{L S T_{\text {man }}-L S T_{\text {min }}} \times 100,
$$

where $L S T_{\text {nor }}$ is the normalized land surface temperature; $L S T_{\min }$ and $L S T_{\max }$ are the minimum and maximum value obtained within the study region, respectively.

Table 2. Estimation of surface emissivity using normalized difference vegetation index (NDVI).

\begin{tabular}{cc}
\hline NDVI & Land Surface Emissivity \\
\hline NDVI $<-0.185$ & 0.995 \\
$-0.185 \leq$ NDVI $<0.157$ & 0.970 \\
$0.157 \leq$ NDVI $\leq 0.727$ & $1.0094+0.047$ Ln(NDVI) \\
NDVI $>0.727$ & 0.990 \\
\hline
\end{tabular}

\section{Results}

\subsection{Urbanization Dynamics Process Assessment in Beijing}

In this study, the annual average NTL-DN value under Beijing's boundary was calculated using Equation (2). The dynamics of urbanization, which was estimated by DMSP/OLS NTL, are shown in Figure 2a. By preprocessing the images, the calibrated annual average NTL-DN value was calculated and is displayed in red. The average NTL-DN increased 1.56 times, from 11.73 in 1992 to 18.28 in 2013 , with an average annual growth rate of $2.16 \%$. Via calibration, we obtained a smooth result of an average NTL-DN trend, which seems more reasonable than the initial NTL data. The total lit number and urban area proportion trends are shown in Figure $2 \mathrm{~b}$ for the selected region. The lit number increased 1.46 times from 14,081 in 1992 to 20,526 in 2013, and the urban area proportion was enhanced 
3.46 times from $4.86 \%$ in 1992 to $16.83 \%$ in 2013. Thus, the lit number increased more rapidly in the first decade than the next; however, the urban area proportion showed an opposite trend where it increased faster in the later decade than before.

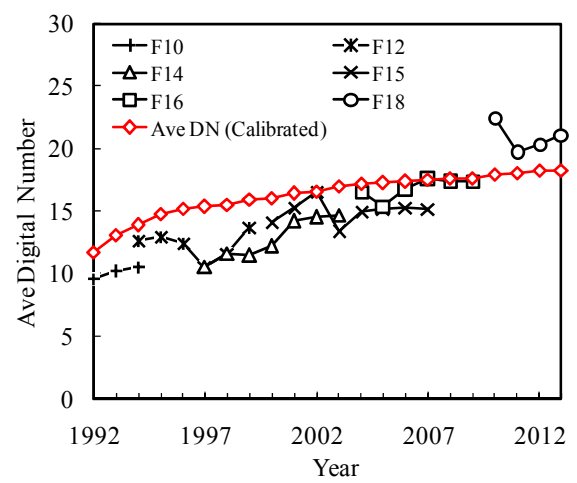

(a)

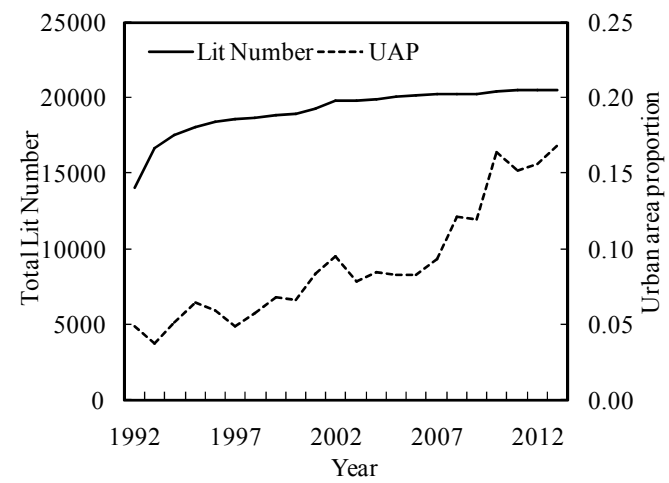

(b)

Figure 2. Dynamics of the urbanization process in Beijing from 1992-2013. (a) Change of annual average nighttime light Digital Number (NTL-DN) in Beijing before and after calibration; and (b) Change of total lit number and urban area proportion in Beijing.

\subsection{Correlation Analysis of the NTL Indicators with Social-Economic Variables}

The relationship between the NTL indicators and social-economic variables was initially examined through a log-linear regression analysis [45]. Representatively, the Average DN-Ln(GDP), Total Lit Number-Ln(GDP) and Urban Area proportion-Ln(GDP) are shown in Figure 3. The regression analysis determined that the DMSP/OLS based indicators were closely related to the GDP of Beijing during the study period. The correlation coefficient of determination of Ln(GDP) with average NTL-DN value, total lit number and urban area proportion were $0.924,0.807$ and 0.815 , respectively, with both showing a close positive correlation. With in-depth analysis through a comparison of the correlation of GDP with NTL indicators, we found that the average NTL-DN had a better correlation than the other two indexes from the approximate increase trends of GDP and Average NTL-DN.

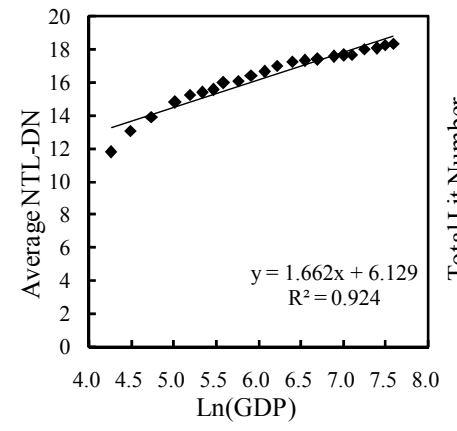

(a)

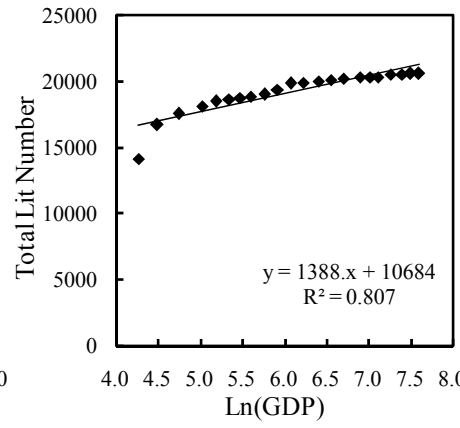

(b)

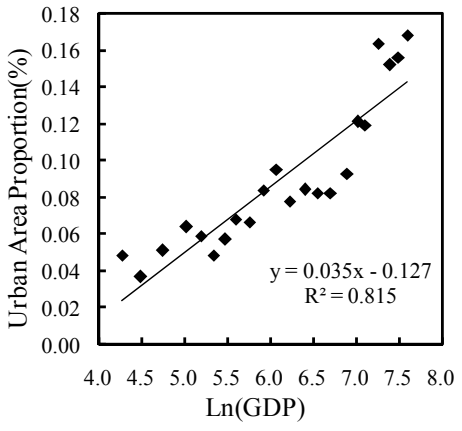

(c)

Figure 3. Scatter plots between the $\operatorname{Ln}(\mathrm{GDP})$ and Defense Meteorological Satellite Program's Operational Line Scanner System (DMSP/OLS) NTL indicators from 1992-2013. (a) Scatter plot between Ln(GDP) and Ave NTL-DN value; (b) Scatter plot between Ln(GDP) and Total Lit Number; and (c) Scatter plot between Ln(GDP) and Urban Area Proportion.

Log-linear regression was then applied between the NTL indicators and all collected social-economic parameters from 1992-1993 to estimate their correlation. Table 3 shows the correlation results, and both average NTL-DN and total lit number and urban area proportion were closely correlated with the social-economic variable. The correlation coefficients of determination of annual 
average NTL-DN value were all above 0.752 with an average value of 0.873 . In particular, the correlation of average NTL-DN with the total consumption of natural gas is among the best, with a determination coefficient of 0.966 . Furthermore, the best correlation of total lit number with social-economics parameters was with the total consumption of natural gas with a determination coefficient of 0.954 , while the average correlation determination coefficient was 0.766 . For the correlation analysis with urban area proportion, the coefficients of determination were all above 0.674 , and held an average value of 0.804 , while the maximum of 0.916 was derived from the population. The result illustrates that DMSP/OLS NTL based urbanization indicators are effective techniques to evaluate and monitor development at city level.

Table 3. Correlation result between DMSP/OLS NTL indicators and social-economic statistics.

\begin{tabular}{|c|c|c|c|c|c|}
\hline \multirow{2}{*}{ Parameter (P) } & \multirow{2}{*}{ Unit } & \multirow{2}{*}{ Increase Rate } & Ave DN & TLN & UAP \\
\hline & & & \multicolumn{3}{|c|}{ Coefficient of Determination $\mathrm{R}^{2}$ with $\operatorname{Ln}(\mathrm{P})$} \\
\hline GDP & Billion & 27.92 & 0.924 & 0.807 & 0.815 \\
\hline Total energy consumption & Million tce & 2.40 & 0.841 & 0.724 & 0.810 \\
\hline Total consumption of natural gas & Billion $\mathrm{m}^{3}$ & 85.30 & 0.966 & 0.954 & 0.674 \\
\hline Total number of civil motor vehicles & Million & 15.16 & 0.895 & 0.771 & 0.840 \\
\hline Average & - & 19.96 & 0.873 & 0.766 & 0.804 \\
\hline
\end{tabular}

\subsection{Spatial Relationship Analysis of DMSP/OLS NTL and Landsat Images}

Figure 4 shows the derived Landsat TM based composite images, NDVI, and normalized LST. Six reflective multispectral bands (Bands 1-5, 7) were used for compositing, and the results are shown in Figure 4a,d. We obtained the spatial pattern of the vegetation coverage in 1995 (Figure 4b) and in 2009 (Figure 4e), an obvious horizontal gradient appeared between the urban area and its surroundings in both 1995 and 2009. The value of the NDVI ranges from $-0.63-0.79$ in 1995, and changes to $-0.93-0.75$ in 2009, and it can be seen that the NDVI changed significantly due to urban expansion during this period. Furthermore, the LST in 1995 and 2009 were normalized (shown in Figure 4c,f, respectively). From the spatial distribution in both years, we concluded that the downtown area had a smaller NDVI value and a larger value of $\mathrm{LST}_{\text {nor }}$, and that through a visual inspection, a surface urban heat island existed within the city.

An analysis based on the $\mathrm{DN}$ value was also conducted to explore the spatial correlation between the DMSP/OLS NTL-DN and Landsat based parameters. The calibrated NTL images are shown in Figure 5 to represent the spatial distribution characteristics of NTL-DN in 1995 (Figure 5a) and 2009 (Figure 5d). Next, the average values of NDVI and $\mathrm{LST}_{\text {nor }}$ in each year were calculated using different NTL-DN values to adopt in the regression analysis with the NTL-DN value. To obtain the relationship between the NDVI and mean NTL-DN in 1995 (Figure 5b) and 2009 (Figure 5e), the results showed that as the value of NTL-DN increased, the trend of mean NDVI decreased, so the coefficient determination of each year reached 0.754 in 1995 and 0.929 in 2009, respectively, which suggests a close negative correlation between the NTL-DN and mean NDVI. The results revealed that in areas with a relatively higher NTL-DN value, the vegetation coverage would be less than in lower NTL-DN value areas. Meanwhile, the correlation between LST $_{\text {nor }}$ and mean NTL-DN in 1995 (Figure 5c) and 2009 (Figure 5f) were established, and in contrast to the mean NDVI, the $\mathrm{LST}_{\text {nor }}$ showed a close positive correlation with determination coefficients reaching 0.651 in 1995 and 0.926 in 2009. In both years, the LST $_{\text {nor }}$ showed an increasing trend along with the rise of the NTL-DN value, which can be used to draw conclusions on how the urbanization level impacts the temperature distribution. The results indicate that for an area with a higher NTL-DN value (that is more urbanized), the mean LST $_{\text {nor }}$ within it would be higher than the surroundings, which provides strong evidence that the surface urban heat island effects are influenced by urban development. 


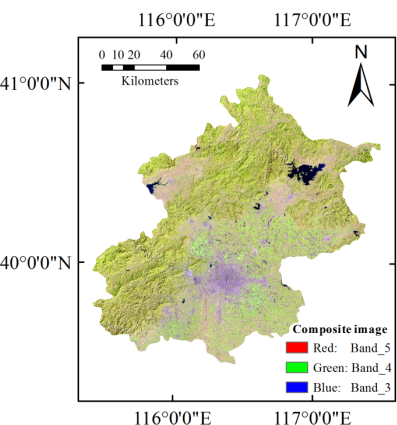

(a)

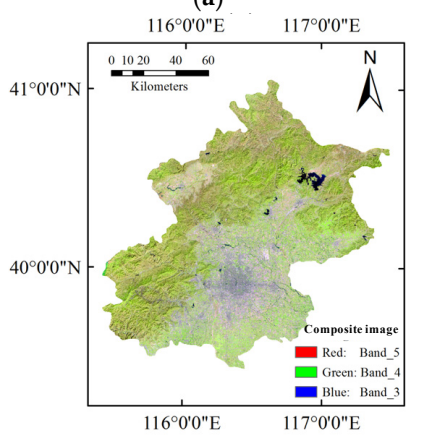

(d)

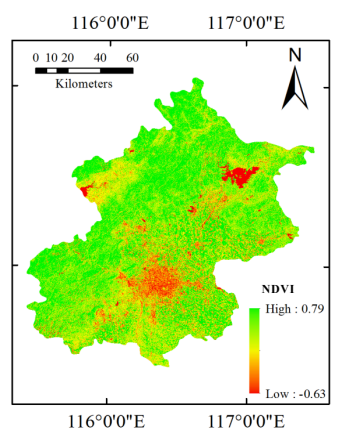

(b)

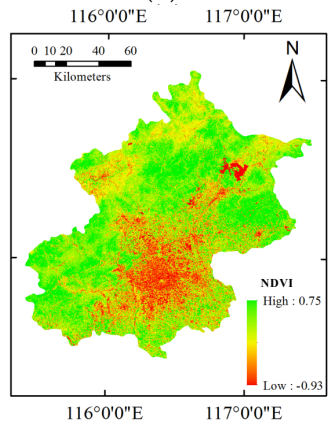

(e)

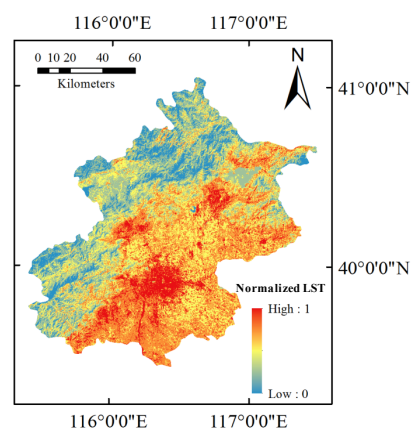

(c)

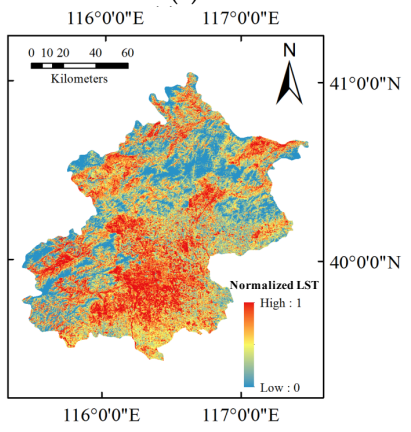

(f)

Figure 4. Distribution map of Landsat thematic mapper (TM) composite image, NDVI, and normalized land surface temperature (LST ${ }_{\text {nor }}$ ). (a) Landsat TM composite image in 1995 (RGB = 543); (b) Distribution map of NDVI in 1995; (c) Distribution map of normalized LST in 1995; (d) Landsat TM composite image in 2009 (RGB = 543); (e) Distribution map of NDVI in 2009; and (f) Distribution map of normalized LST in 2009.
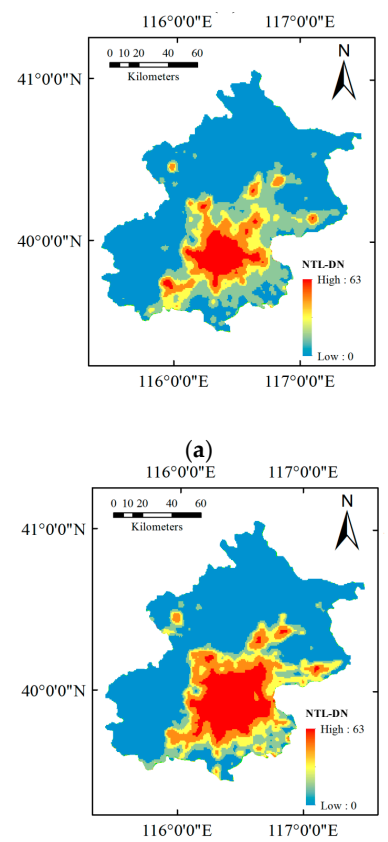

(d)

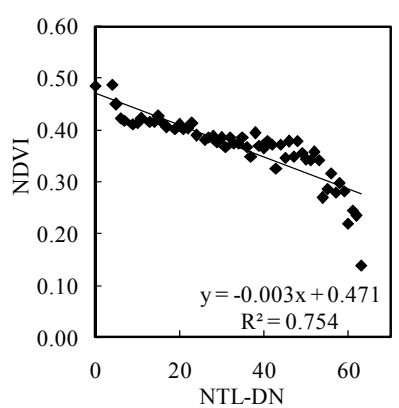

(b)

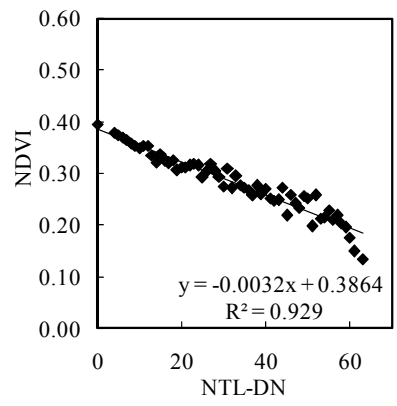

(e)

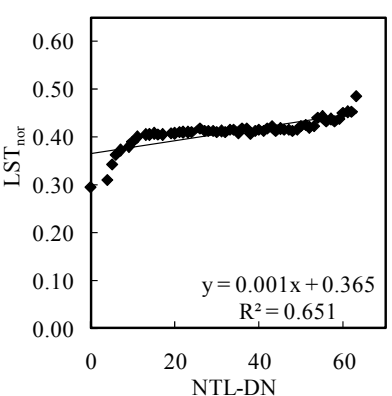

(c)

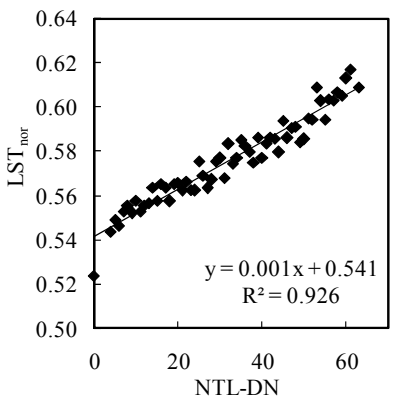

(f)

Figure 5. Spatial correlation analysis of NTL-DN and Landsat images: (a) Distribution map of NTL-DN in 1995; (b) Scatter plots between the NTL-DN and NDVI in 1995; (c) Scatter plots between the NTL-DN and LST $_{\text {nor }}$ in 1995; (d) Distribution map of NTL-DN in 2009; (e) Scatter plots between the NTL-DN and NDVI in 2009; and (f) Scatter plots between the NTL-DN and LST nor $_{2009 .}$ 


\subsection{Spatial-Temporal Comparison Analysis in 1995 and 2009}

To investigate the influence of SUHI driven by urban development, the images of NTL-DN, NDVI and $\mathrm{LST}_{\text {nor }}$ from two years was composited to obtain the difference between 1995 and 2009. Next, a spatial-temporal comparison analysis was carried out to explore the variation in vegetation coverage situation and the SUHI effects from the urbanization process revealed by NTL images. Figure 6a shows the spatial pattern of the NTL-DN difference between the two years through visual assessment, with the NTL-DN of the previous downtown area remaining unchanged, whereas the surrounding areas had an obvious increase in NTL-DN value, which matched the urban sprawl and expansion in Beijing during this period. With the extraction of the NTL-DN difference, correlation analysis was then carried with using the NDVI difference during the study period (Figure 6b). The NDVI difference showed a negative correlation with the NTL-DN difference, where the determination coefficient reached 0.681, and the correlation was more obvious in the range where theNTL-DN difference had a positive value. The result suggested that the urbanization process would reduce vegetation coverage, given that urban sprawl changed the vegetation area into impervious surfaces. Another correlation analysis was

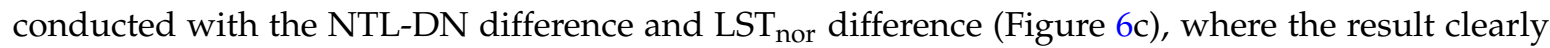
demonstrated a positive correlation between the two parameters, where the coefficient of determination was 0.664 . Areas with a higher value of NTL-DN difference, which represent faster urbanization zones, and the $\mathrm{LST}_{\text {nor }}$ difference showed a tendency to increase. The faster the development in the area, the higher the increase of $\mathrm{LST}_{\text {nor }}$ difference. Additionally, the result further illustrated how urbanization affected the SUHI quantitatively over time in the city.

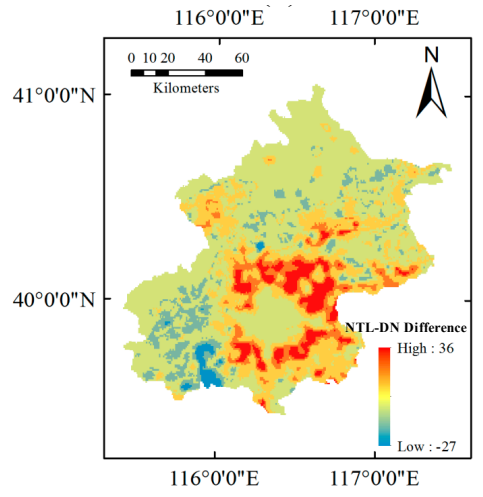

(a)

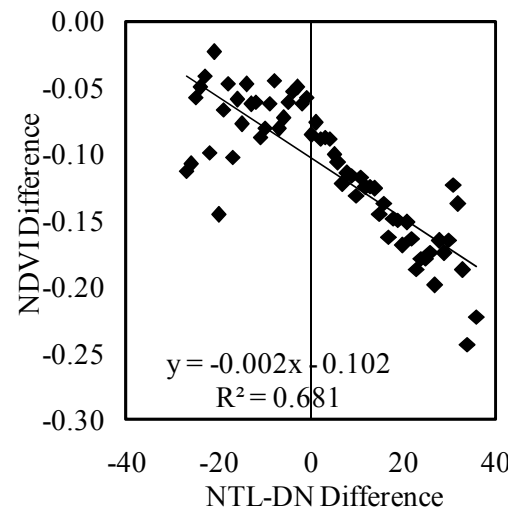

(b)

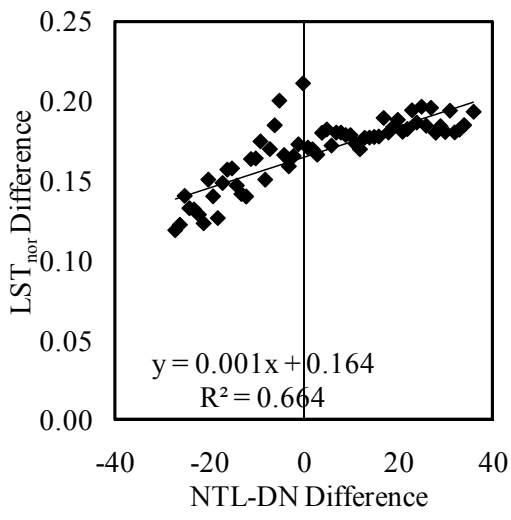

(c)

Figure 6. Spatial comparison analysis of NTL-DN and Landsat images: (a) Distribution map of the NTL-DN difference between 1995 and 2009; (b) Scatter plots between the NTL-DN difference and NDVI change between 1995 and 2009; and (c) Scatter plots between the NTL-DN difference and LST nor change between 1995 and 2009.

\subsection{Urban Heat Island Analysis}

Figure 7a,d show the spatial pattern of urban heat island zones in 1995 and 2009, respectively. The average normalized LST was calculated within each NTL pixel to indicate the surface temperature distribution. Next, choropleth maps were produced based on the classification scheme of standard deviation, in which the first-class data values were greater than one standard deviation above the mean, and second-class values were between the mean and one standard deviation above the mean, and so on $[39,46]$. Thus, the area was divided into four categories: heat island zone, sub-heat island zone, medium temperature zone, and low temperature zone. In both 1995 and 2009, the urban heat island zone was obviously assembling in the city center, and the heat island zone had a large expansion from all directions. Figure $7 \mathrm{~b}$,e shows the area proportions and the average $\mathrm{LST}_{\text {nor }}$ for the four categories, where the percentage of urban heat island zone increased from $9.3 \%$ in 1995 to 
$21.7 \%$ in 2009; the sub-heat island zone had no significant change from $29.3 \%$ to $28.6 \%$; and both the medium temperature and low temperature zones had a decrease of area proportion, especially the low temperature area which decreased from $44.0 \%-35.7 \%$. These changes indicated an adverse effect on the thermal environment through the urbanization process from 1995-2009, and that the urban heat island zone occupied a higher proportion than before.

To better understand the relationship between the NTL-DN, NDVI and UHI zones, the statistics of average NTL-DN and NDVI in different UHI zones were obtained by super-imposing the heat island maps with images of NTL and NDVI. From Figure 7c,f, we obtained the characteristics of average NTL-DN and NDVI of each zone in 1995 and 2009. In both 1995 and 2009, the value of average NTL-DN presented a rising gradient from the low temperature zone to the heat island zone, whereas the NDVI showed a downward trend. The urban heat island zone held the highest average NTL-DN value of 58.26 in 1995, and the average values in the other three zones were 35.40, 25.73 and 10.03, respectively. In 2009, the average NTL-DN values in the four zones were 12.65, 24.95, 41.26 and 54.34, respectively, from the low temperature zone to the heat island zone. The results illustrated the spatial correlation between the surface temperature and the NTL images. The urban heat island zones located in more developed areas, the sub-heat island and medium temperature zones took second and third place, and the low temperature zones were mainly located in the western and northern parts of the city where most of the area was covered with mountains and forests that have lower NTL-DN values than downtown. Furthermore, in both 1995 and 2009, the low temperature zones had the highest value of NDVI, whereas the urban heat island zones had the lowest. These results showed a positive correlation between the proportion of green space and their cooling effects.

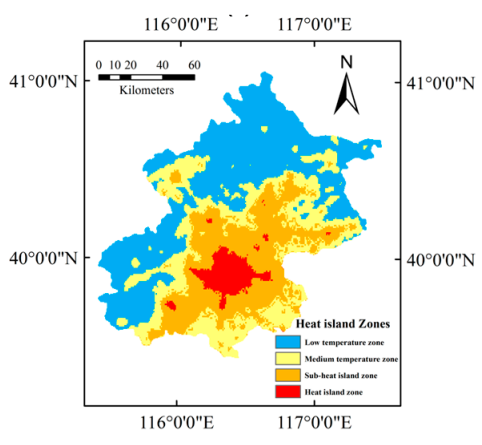

(a)

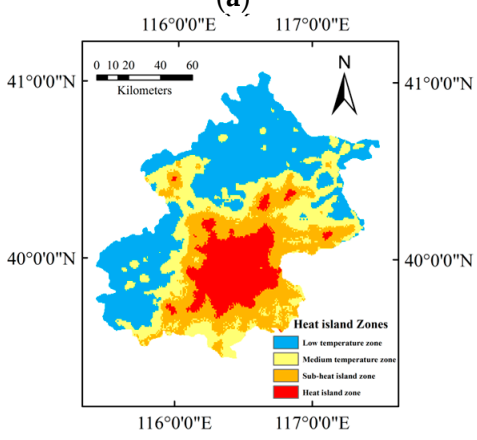

(d)

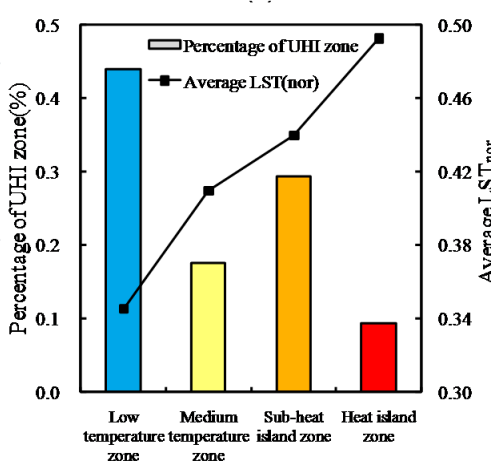

(b).

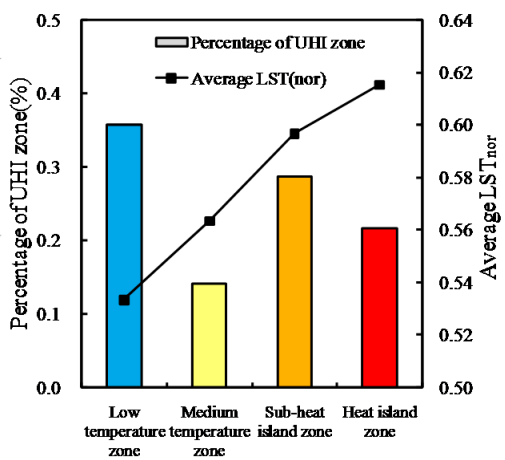

(e)

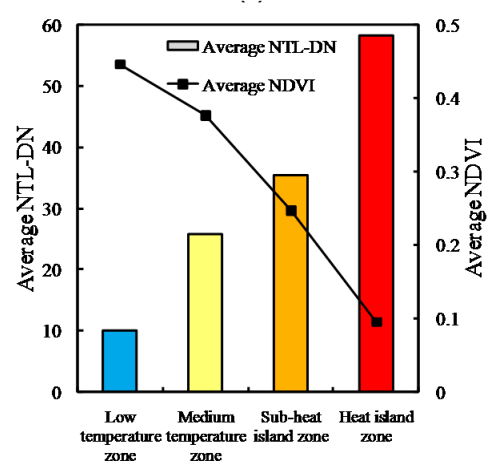

(c)

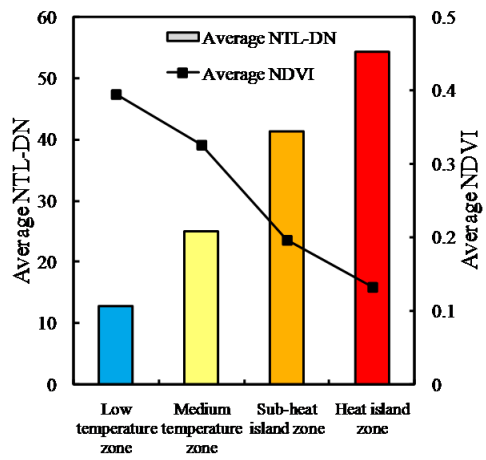

(f)

Figure 7. Heat island analysis results with NTL-DN and Landsat images: (a) Spatial pattern of heat island zones in 1995; (b) Proportions of urban heat island (UHI) zones and average LST nor in 1995; (c) Average NTL-DN and average NDVI of UHI zones in 1995; (d) Spatial pattern of heat island zones in 2009; (e) Proportions of UHI zone and average LST nor in 2009; and (f) Average NTL-DN and average NDVI of UHI zones in 2009. 


\section{Discussion}

\subsection{Result Analysis}

Urban systems do not naturally develop, and are always affected by human activities [2]. As a remote sensing database, which is closely related to anthropogenic activities, the time series DMSP/OLS images provide a consistent and timely measure to estimate social-economic dynamics and changes [7]. In this study, the calibrated annual NTL-DN value from 1992-2013 was used to reflect the average urbanization level of the city in that year, as a global index to evaluate the development level of Beijing. The results show that the human activities observed via satellite were consistent with the official statistical data. The average value of the correlation coefficient of determination between the three remote sensing based indexes with the seven statistical indicators reached $0.873,0.766$ and 0.804, respectively. Compared with the other two indicators, the coefficient of total lit number was a little less, most likely due to the characteristics of urbanization pattern in Beijing. The lit number increased quickly in the few years from 1992 as a fast period of urban sprawl, then slowed down and the urbanization level in the expanded area developed rapidly instead of a continuous outward expansion. In contrast, the social-economic statistics showing a sustained and rapid growth during the period led to less correlation with the total lit number than the other two parameters. The urbanization situation in the study period was confirmed by three fields: the average NTL-DN value (within the administrative boundaries of Beijing) presented an average urbanization situation of the city in each year; the total lit number was an indicator from which the number of pixels lit by human activities could be obtained; and the urban area proportion revealed the cases of urban area sprawl during the study period.

Land surface temperature observations acquired from remote sensing technologies such as MODIS and Landsat were applied to assess the UHI, and to analyze the relationship between surface temperature and land use and land cover (LULC) in urban areas [39]. Nighttime light imagery derived from DMSP/OLS has been widely used for detecting human settlement, dynamic mapping of urban areas and exploring the spatial urban sprawl $[8,9]$. For the use of these two kinds of databases in combination, Liao et al. [19] found that the DMSP/OLS NTL based energy consumption has a significantly positive correlation with the nighttime SUHI, the results indicated that the anthropogenic heat released from energy consumption is an important contributor to the urban thermal environment [19]. In addition, researchers also found that urban patterns extracted using DMSP/OLS were consistent with those extracted using Landsat products [33]. However, the number of studies estimating the correlation between the Landsat based LST with nighttime imagery from the DMSP/OLS are rare and limited. This paper proposed a new way of integrating different remote sensing data to explore the performance of NDVI and LST by Landsat TM images under different values of NTL-DN from DMSP/OLS imagery. The results clearly showed that the spatial pattern of $\mathrm{LST}_{\text {nor }}$ and NDVI correlated with the pattern of urbanization level in 1995 and 2009. Moreover, by spatial comparison analysis between the two years, the spatial pattern of LST $_{\text {nor }}$ increase and NDVI decrease also proved to be closely related to the change of urbanization level index developed from the nighttime imagery. Furthermore, the urban heat island zone maps presented the spatial pattern of surface temperature distribution, where the results revealed that the urban heat island zone held higher NTL-DN values and lower NDVI than other zones.

\subsection{Limitations and Potential Improvement}

Although the DMSP/OLS time series was successfully adopted to explore urbanization dynamics and analyze the UHI situation in Beijing, there are some sources of uncertainty in this study. First, for the inter-calibration, Yichun City of Heilongjiang Province was selected as the reference region even though the area had little change of NTL during the study period; however, the inappreciable development of the city reduced the accuracy of the calibration. Second, for the calculation of the urban area proportion, we adopted the threshold technique provided by Gao et al. [35], where the 
thresholds for urban area extraction were determined for several periods rather than every year, which is one of the influence factors for the accuracy of extraction. Third, the calculation of the normalized LST contained limitations. The normalization relied heavily on the extreme values within the region; however, as most of the pixel values ranged in the middle area between the maximum and minimum values, normalization reduced the sensitivity of the analysis. Fourth, in this study, four temporal Landsat time series image data were used to characterize and quantify the spatiotemporal patterns of NDVI and LST $_{\text {nor, }}$, and correlation analysis was carried out with the annual value of NTL-DN from DMSP/OLS. As this would have created limitations for the correlation analysis between the annual composites of DMSP/OLS and Landsat images, it was necessary to develop new methods that could be applied effectively to detect the annual value of NDVI and LST $_{\text {nor }}$ using time series image data.

\section{Conclusions}

This study developed the annual average NTL-DN value, total lit number and urban area proportion to investigate the spatial-temporal urbanization progress in Beijing from 1992-2013. Our results offer quantitative measures for evaluating the urbanization level from three aspects over the period, and provide a new way to explore the urbanization process that is relatively accurate and comprehensive.

From both the DMSP/OLS NTL indicators and the social-economic statistics data, the results showed that Beijing experienced continued and rapid urbanization during the study period. Log-linear regression analysis between the two kinds of data sets showed close correlation with each other, with the average value of determination coefficient of annual average NTL-DN value, total lit number and urban area proportion reached $0.873,0.766$ and 0.804 , respectively.

From the distribution map of NDVI and LST $_{\text {nor, }}$ we found that the downtown area had less vegetation coverage and a higher $\mathrm{LST}_{\text {nor }}$. Furthermore, according to the correlation analysis with

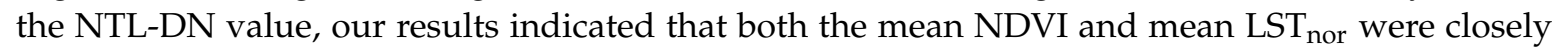
correlated with the NTL data. The NDVI and LST $_{\text {nor }}$ showed a tendency of decreasing and increasing, respectively, along with the growth of the NTL-DN value. The study proposed a new way of integration using different remote sensing data to explore the urban heat island effects by Landsat TM images and DMSP/OLS imagery. The results provided quantitative measurements to estimate the vegetation coverage situation and surface temperature distribution, which are significant for the eco-environment assessment of the city.

The study also explored the spatial-temporal difference of NDVI and LST nor $_{\text {along with the }}$ urbanization process derived from NTL data in 1995 and 2009. Along with the rapid urbanization of the city, the original land use turned into urban land, which is largely composed of an impervious surface [23]. The correlation analysis result between the NTL-DN difference with a change in NDVI and LST $_{\text {nor }}$ showed a close relationship, which provided strong evidence of how the urbanization process affects the thermal environment and vegetation coverage over time. In addition, by zonal classification, we also found that the urban heat island zone occupied a higher proportion than before by a growth rate of $12.4 \%$, and that the average NTL-DN and NDVI showed an obvious gradient from the low temperature zone to heat island zone. Our results illustrated the spatial correlation between the surface temperature and the urban development situation, as well as the cooling effects of the green space, which have important implications for urban planning to mitigate the SUHI effects.

Acknowledgments: We would like to express our respect and gratitude to the anonymous reviewers and editors for their valuable comments and suggestions on improving the quality of the paper.

Author Contributions: Chen Wei conceived and designed the study and methods. Chen Wei, Zhang Yao and Pengwang Chongyu made substantial contributions to acquisition, analysis, and interpretation of the data. Chen Wei wrote the first draft of the article. Zhang Yao, Pengwang Chongyu and Gao Weijun reviewed and edited the first draft. All authors read and approved the submitted manuscript, agreed to be listed, and accepted the version for publication.

Conflicts of Interest: The authors declare no conflict of interest. 


\section{References}

1. United Nations. World Urbanization Prospects: The 2007 Revision; United Nations: New York, NY, USA, 2008.

2. United Nations. World Urbanization Prospects: The 2014 Revision; United Nations: New York, NY, USA, 2008.

3. Shao, M.; Tang, X.; Zhang, Y.; Li, W. City clusters in China: Air and surface water pollution. Front. Ecol. Environ. 2006, 4, 353-361. [CrossRef]

4. Chan, C.K.; Yao, X. Air pollution in mega cities in China. Atmos. Environ. 2008, 42, 1-42. [CrossRef]

5. Zhu, H.M.; You, W.H.; Zeng, Z.F. Urbanization and CO2 emissions: A semi-parametric panel data analysis. Econ. Lett. 2012, 117, 848-850. [CrossRef]

6. Zhou, L.M.; Dickinson, R.E.; Tian, Y.H.; Fang, J.Y.; Li, Q.X.; Kaufmann, R.K.; Tucker, C.J.; Myneni, R.B. Evidence for a significant urbanization effect on climate in China. Proc. Natl. Acad. Sci. USA 2004, 101, 9540-9544. [CrossRef] [PubMed]

7. Ma, T.; Zhou, C.H.; Pei, T.; Haynie, S.; Fan, J.F. Quantitative estimation of urbanization dynamics using time series of DMSP/OLS nighttime light data: A comparative case study from China's cities. Remote Sens. Environ. 2012, 124, 99-107. [CrossRef]

8. Elvidge, C.D.; Baugh, K.E.; Kihn, E.A.; Kroehl, H.W.; Davis, E.B. Mapping city lights with nighttime data from the DMSP operational linescan system. Eng. Remote Sens. 1997, 63, 727-734.

9. Sutton, P.C. A scale-adjusted measure of "urban sprawl" using nighttime satellite imagery. Remote Sens. Environ. 2003, 86, 353-369. [CrossRef]

10. Dobson, J.E.; Brlght, E.A.; Coleman, P.R.; Durfee, R.C.; Worley, B.A. Landscan: A global population database for estimating populations at risk. Photogramm. Eng. Remote Sens. 2000, 66, 849-857.

11. Sutton, P.; Roberts, D.; Elvidge, C.; Melj, H. A comparison of nighttime satellite imagery and population density for the continental united states. Photogramm. Eng. Remote Sens. 1997, 63, 1303-1313.

12. Sutton, P.C.; Costanza, R. Global estimates of market and non-market values derived from nighttime satellite imagery, land cover, and ecosystem service valuation. Ecol. Econ. 2002, 41, 509-527. [CrossRef]

13. He, C.; Ma, Q.; Liu, Z.; Zhang, Q. Modeling the spatiotemporal dynamics of electric power consumption in mainland China using saturation-corrected DMSP/OLS nighttime stable light data. Int. J. Digit. Earth 2013, 7, 993-1014. [CrossRef]

14. Doll, C.N.H.; Muller, J.-P.; Elvidge, C.D. Night-time imagery as a tool for global mapping of socioeconomic parameters and greenhouse gas emissions. Ambio 2000, 29, 157-162. [CrossRef]

15. Xu, T.; Ma, T.; Zhou, C.; Zhou, Y. Characterizing spatio-temporal dynamics of urbanization in China using time series of DMSP/OLS night light data. Remote Sens. 2014, 6, 7708-7731. [CrossRef]

16. Luck, M.; Wu, J. A gradient analysis of urban landscape pattern: A case study from the Phoenix metropolitan region, Arizona, USA. Landsc. Ecol. 2002, 17, 327-339. [CrossRef]

17. Voogt, J.A.; Oke, T.R. Thermal remote sensing of urban climates. Remote Sens. Environ. 2003, 86, 370-384. [CrossRef]

18. Streutker, D.R. A remote sensing study of the urban heat island of Houston, Texas. Int. J. Remote Sens. 2002, 23, 2595-2608. [CrossRef]

19. Liao, W.; Liu, X.; Wang, D.; Sheng, Y. The impact of energy consumption on the surface urban heat island in China's 32 major cities. Remote Sens. 2017, 9, 250. [CrossRef]

20. Noi, P.T.; Kappas, M.; Degener, J. Estimating daily maximum and minimum land air surface temperature using MODIS land surface temperature data and ground truth data in Northern Vietnam. Remote Sens. 2016, 8, 1002. [CrossRef]

21. Nichol, J.E. A GIS-based approach to microclimate monitoring in Singapore's high-rise housing estates. Photogramm. Eng. Remote Sens. 1994, 60, 1225-1232.

22. Weng, Q.; Lu, D.; Liang, B. Urban surface biophysical descriptors and land surface temperature variations. Photogramm. Eng. Remote Sens. 2006, 72, 1275-1286. [CrossRef]

23. Yuan, F.; Bauer, M.E. Comparison of impervious surface area and normalized difference vegetation index as indicators of surface urban heat island effects in Landsat imagery. Remote Sens. Environ. 2007, 106, 375-386. [CrossRef]

24. Carlson, T.N.; Gillies, R.R.; Perry, E.M. A method to make use of thermal infrared temperature and NDVI measurements to infer surface soil water content and fractional vegetation cover. Remote Sens. Rev. 1994, 9, 161-173. [CrossRef] 
25. Goward, S.N.; Xue, Y.; Czajkowski, K.P. Evaluating land surface moisture conditions from the remotely sensed temperature/vegetation index measurements: An exploration with the simplified simple biosphere model. Remote Sens. Environ. 2002, 79, 225-242. [CrossRef]

26. Lo, C.P.; Quattrochi, D.A.; Luvall, J.C. Application of high-resolution thermal infrared remote sensing and GIS to assess the urban heat island effect. Int. J. Remote Sens. 1997, 18, 287-304. [CrossRef]

27. Deng, X.; Huang, J.; Rozelle, S.; Uchida, E. Growth, population and industrialization and urban land expansion of China. J. Urban Econ. 2008, 63, 96-115. [CrossRef]

28. Zhang, K.H.; Song, S. Rural-urban migration and urbanization in China: Evidence from time-series and cross-section analyses. China Econ. Rev. 2003, 14, 386-400. [CrossRef]

29. National Bureau of Statistics of the People's Republic of China. Available online: http://www.stats.gov.cn/ (accessed on 25 October 2016).

30. Takahashi, K.I.; Terakado, R.; Nakamura, J.; Daigo, I.; Matsuno, Y.; Adachi, Y. In-use stock of copper analysis using satellite nighttime light observation data. Mater. Trans. 2009, 50, 1871-1874. [CrossRef]

31. Elvidge, C.D.; Sutton, P.C.; Baugh, K.E.; Ziskin, D.; Ghosh, T.; Anderson, S. National trends in satellite observed lighting: 1992-2012. Available online: http://ngdc.noaa.gov/eog/dmsp/download_national_ trend.html (accessed on 25 October 2016).

32. Elvidge, C.D.; Ziskin, D.; Bough, K.E.; Tuttle, B.T.; Ghosh, T.; Pack, D.W.; Erwin, E.H.; Zhizhin, M. A fifteen year record of global natural gas flaring derived from satellite data. Energies 2009, 2, 595-622. [CrossRef]

33. Liu, Z.; He, C.; Zhang, Q.; Huang, Q.; Yang, Y. Extracting the dynamics of urban expansion in China using DMSP-OLS nighttime light data from 1992 to 2008. Landsc. Urban Plan. 2012, 106, 62-72. [CrossRef]

34. Henderson, M.; Yeh, E.T.; Gong, P.; Elvidge, C.D.; Baugh, K.E. Validation of urban boundaries derived from global night-time satellite imagery. Int. J. Remote Sens. 2003, 24, 595-609. [CrossRef]

35. Gao, B.; Huang, Q.X.; He, C.Y.; Ma, Q. Dynamics of Urbanization Levels in China from 1992 to 2012 : Perspective from DMSP/OLS Nighttime Light Data. Remote Sens. 2015, 7, 1721-1735. [CrossRef]

36. USGS Earth Explorer. Available online: http:/ / earthexplorer.usgs.gov/ (accessed on 25 October 2016).

37. Chander, G.; Groeneveld, D.P. Intra-annual NDVI validation of the Landsat 5 TM radiometric calibration. Int. J. Remote Sens. 2009, 30, 1621-1628. [CrossRef]

38. Landsat 7 Science Data Users Handbook. Available online: http:/ /landsat.gsfc.nasa.gov/landsat-7-sciencedata-users-handbook/ (accessed on 25 October 2016).

39. Qinghao, W.; Dengsheng, L.; Schubring, J. Estimation of land surface temperature-vegetation abundance relationship for urban heat island studies. Remote Sens. Environ. 2004, 89, 467-483.

40. Artis, D.A.; Carnahan, W.H. Survey of emissivity variability in thermography of urban areas. Remote Sens. Environ. 1982, 12, 313-329. [CrossRef]

41. Markham, B.L.; Barker, J.L. Spectral characterization of the LANDSAT thematic mapper sensors. Int. J. Remote Sens. 1985, 6, 697-716. [CrossRef]

42. Van de Griend, A.A.; Owe, M. On the relationship between thermal emissivity and the normalized difference vegetation index for natural surfaces. Int. J. Remote Sens. 2003, 14, 1119-1131. [CrossRef]

43. Zhang, J.; Wang, Y.; Li, Y. A C++ program for retrieving land surface temperature from the data of Landsast TM/ETM Band 6. Comput. Geosci. 2006, 32, 1796-1805. [CrossRef]

44. Xu, H.Q.; Chen, B.Q. Remote sensing of the urban heat island and its changes in Xiamen City of SE China. J. Environ. Sci. 2004, 16, 276-281.

45. Oke, T.R. City size and the urban heat island. Atmos. Environ. 1973, 7, 769-779. [CrossRef]

46. Smith, R.M. Comparing traditional methods for selecting class intervals on choropleth maps. Prof. Geogr. 1986, 38, 62-67. [CrossRef]

(C) 2017 by the authors. Licensee MDPI, Basel, Switzerland. This article is an open access article distributed under the terms and conditions of the Creative Commons Attribution (CC BY) license (http:/ / creativecommons.org/licenses/by/4.0/). 